\title{
REAS3: Monte Carlo simulations of radio emission from cosmic ray air showers using an "end-point" formalism
}

\author{
M. Ludwig ${ }^{a, *}$, T. Huege ${ }^{\mathrm{b}}$ \\ ${ }^{a}$ Karlsruher Institut für Technologie, Institut für Experimentelle Kernphysik - \\ Campus Süd, 76128 Karlsruhe, Germany \\ ${ }^{\mathrm{b}}$ Karlsruher Institut für Technologie, Institut für Kernphysik - Campus Nord, \\ Postfach 3640, 76021 Karlsruhe, Germany
}

\begin{abstract}
In recent years, the freely available Monte Carlo code REAS for modelling radio emission from cosmic ray air showers has evolved to include the full complexity of air shower physics. However, it turned out that in REAS2 and all other time-domain models which calculate the radio emission by superposing the radiation of the single air shower electrons and positrons, the calculation of the emission contributions was not fully consistent. In this article, we present a revised implementation in REAS3, which incorporates the missing radio emission due to the variation of the number of charged particles during the air shower evolution using an "end-point formalism". With the inclusion of these emission contributions, the structure of the simulated radio pulses changes from unipolar to bipolar, and the azimuthal emission pattern becomes nearly symmetric. Remaining asymmetries can be explained by radio emission due to the variation of the net charge excess in air showers, which is automatically taken into account in the new implementation. REAS3 constitutes the first self-consistent time-domain implementation based on single particle emission taking the full complexity of air shower physics into account, and is freely available for all interested users.
\end{abstract}

Key words: cosmic rays, extensive air showers, electromagnetic radiation from moving charges, computer modeling and simulation, radio emission, endpoint formalism

\footnotetext{
* Corresponding author.

Email address: marianne.ludwig@kit.edu (M. Ludwig).
} 


\section{Introduction}

High energy cosmic rays initiate extensive air showers when entering the Earth's atmosphere. The electromagnetic component of the air shower produces radio emission which can be measured to study the characteristics of the cosmic rays. Radio detector arrays like LOPES [1], 2] and CODALEMA [3], 4] have verified that radio emission in the air is dominated by a geomagnetic effect, and they have studied correlations of the radio signal with shower parameters in great detail. With AERA [5] within the Pierre Auger Observatory [6] and LOFAR [7], radio detection will be applied on larger scales and new "super-hybrid" techniques for measuring cosmic ray air showers will be employed. To understand the measurements and to learn more about the physics of cosmic rays using radio signals from air showers, a solid theoretical understanding of the radio emission process is needed. Presently, two major approaches exist, both of which are mainly based on geomagnetic effects [8]. On the one hand, there is a model based on the calculation of emission from the deflection of single particles in the geomagnetic field which was mainly investigated by Huege et al. [9], [10], [11], [12], in particular with the implementation in the REAS Monte Carlo code. The treatment of the radio emission with Monte Carlo techniques makes it straight-forward to couple it with detailed Monte Carlo air shower simulations. In case of REAS, this is done with CORSIKA [13] in which the needed particle distributions are histogrammed by COAST [12. It is interesting to note that the original frequency-domain calculation of this implementation predicted spectra decaying to zero at small frequencies [9], the time-domain implementation in the simulation code REAS, however, hitherto predicted spectra levelling off at a finite field strength for small frequencies, leading to essentially unipolar pulses [11], [12].

On the other hand, the macroscopic description of geomagnetic radiation (MGMR) developed by Scholten et al. [14] constitutes a modern implementation of the approach for radio emission modelling by Kahn and Lerche in 1966 [15]. Due to the Lorentz force, moving electrons and positrons are separated in the Earth's magnetic field. In MGMR, this is described by a time-variable net electric current in the electron-positron plasma, which is moving through the atmosphere with approximately the speed of light. The emission of an electromagnetic pulse is caused by these time-dependent transverse currents. Due to the dependence on the evolution of the charged particles in the shower with time, the MGMR model predicts a bipolar structure of the pulse shape [14].

The differences in the results of both models were studied and led to the conclusion that at least one model was not complete. The main reason for the different results of both approaches was that in REAS2 (and all other timedomain approaches based on single particle emission, e.g. [16], [17]), emission due to the variation of the number of charged particles within the shower was not considered. A detailed discussion of this issue can be found in [18] to which 
we kindly refer the reader for further details. To correct the implementation in REAS, these missing radiation contributions had to be taken into account. It should be stressed that the resulting implementation, based on the "endpoint" formalism, consistently describes the radiation of the complete underlying particle motion, not just "synchrotron"-like emission from the particle acceleration (see [19] for a discussion of the universal nature of the end-point formalism).

\section{Simulation algorithm of REAS}

From REAS2 to REAS3, the simulation algorithm is unchanged and the air shower information is provided by CORSIKA and COAST in the same way as before. Therefore, we here only give a short overview of the technical implementation and for details, we kindly refer the interested reader to [12].

First, the shower is simulated with CORSIKA using the air shower parameters of interest (such as primary energy, magnetic field, mass of primary, incoming direction, etc.). Using COAST, the information of the electrons and positrons is saved in histograms. These histograms contain information about the atmospheric depth of the particle, the particle arrival time, the lateral distance of the particle from the shower axis, the particle energy and the particle momentum direction. In the next step, REAS is generating individual electrons and positrons randomly according to the histogrammed distributions. These particles are then tracked analytically in the geomagnetic field. Note that the trajectories of the REAS simulation do not represent real trajectories of particles, i.e. one long particle trajectory is represented by an ensemble of several shorter, unrelated trajectories in the code. The length of the trajectories is determined by a parameter $\lambda$ which is explained in chapter 5 .

In REAS3, the trajectory is distributed symmetrically around the position at which the particle is generated.

\section{Contributions due to charge variation}

In REAS2 [12], the radiation of single particles of an air shower is calculated as

$$
\vec{E}(\vec{x}, t)=e\left[\frac{\vec{n}-\vec{\beta}}{\gamma^{2}(1-\vec{\beta} \cdot \vec{n})^{3} R^{2}}\right]_{\text {ret }}+\frac{e}{c}\left[\frac{\vec{n} \times[(\vec{n}-\vec{\beta}) \times \dot{\vec{\beta}}]}{(1-\vec{\beta} \cdot \vec{n})^{3} R}\right]_{\text {ret }},
$$

where $e$ indicates the particle charge, $\vec{\beta}=\vec{v}(t) / c$ is given by the particle velocity, $R(t)=|\vec{R}(t)|$ describes the vector between particle and observer position, 
$\vec{n}(t)=\vec{R}(t) / R(t)$ is the line-of-sight direction between particle and observer, and $\gamma$ is the Lorentz factor of the particle. The index "ret" means that the equation needs to be evaluated in retarded time.

The electrons and positrons emit radiation continuously along their track. To get a consistent description of radio emission in air showers, however, not only contributions due to the deflection of the particles in the magnetic field have to be taken into account, but also contributions due to the variation of the number of charged particles.

REAS2 treats radiation processes only along the trajectories, but not at the end or the beginning of trajectories. Strictly speaking, this is equivalent to the situation that the particle arrives with the velocity $v \approx c$ given by CORSIKA, enters the Earth's magnetic field where it is deflected and describes a short curved track and finally flies out of the influence of the magnetic field still with a velocity $v \approx c$. Figure 1 shows a sketch for such a particle trajectory. It is

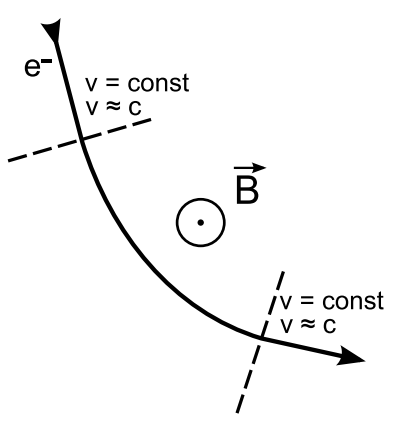

Fig. 1. Sketch of the the trajectory how it was implemented in REAS2.

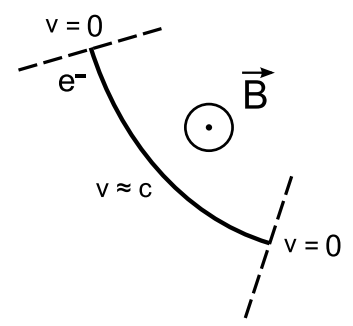

Fig. 2. Sketch of the the trajectory with start- and end-point as needed for a consistent description of radio emission from EAS.

obvious that this is not describing the real situation in an air shower. Consequently, to complement the description in the Monte Carlo code, radiation at the beginning and at the end of each particle trajectory has to be calculated. If at a given atmospheric depth more particle trajectories start than end, i.e. the number of charged particles grows, this results in a net contribution. The same is true if the number of charged particles declines, i.e. more particle tracks end than start. Note that a net contribution occurs as well due to the change of the geometrical distribution caused by the spatial separation of the charged particles.

In REAS3, a particle is treated as if it was created at rest and became "instantaneously" accelerated to $v \approx c$, flew on a short curved track through the Earth's magnetic field and got decelerated to rest again (see figure 21). The acceleration process at the injection as well as the removal of an electron or positron takes place on time scales short in comparison with the frequencies of interest $\left(\nu_{\text {observed }} \leq 100-1000 \mathrm{MHz}\right)$, i.e. $\delta t \ll \frac{1}{\nu_{\text {observed }}}$. Hence, only the time-averaged process is of interest, which will give a discrete contribution in contrast to the continuous emission along the curved particle trajectory. To 
calculate contributions for the start-point, we consider the far field term of the general radiation equation and integrate over the injection time $\delta t$. The "static" term of the radiation formula (1), the velocity field, can be neglected, because the "radiation" term completely dominates the signal for distances $R$ relevant in practical applications. The relation for the retarded time used for transforming the integral from $\mathrm{d} t$ to $\mathrm{d} t^{\prime}$ is derived from $t^{\prime}=t-R\left(t^{\prime}\right) / c$. With $\mathrm{d} t=(1-\vec{\beta} \cdot \vec{n}) \mathrm{d} t^{\prime}$ the integral is solved as shown in the following calculation:

$$
\begin{aligned}
\int \vec{E}(\vec{x}, t) \mathrm{d} t & =\frac{e}{c} \int_{t_{0}}^{t_{1}}\left|\frac{\vec{n} \times[(\vec{n}-\vec{\beta}) \times \dot{\vec{\beta}]}}{(1-\vec{\beta} \cdot \vec{n})^{3} R}\right|_{r e t} \mathrm{~d} t=\frac{e}{c R} \int_{t_{0}^{\prime}}^{t_{1}^{\prime}} \frac{\vec{n} \times[(\vec{n}-\vec{\beta}) \times \dot{\vec{\beta}}]}{(1-\vec{\beta} \cdot \vec{n})^{2}} \mathrm{~d} t^{\prime} \\
& =\frac{e}{c R} \int_{t_{0}^{\prime}}^{t_{1}^{\prime}} \frac{\mathrm{d}}{\mathrm{d} t}\left(\frac{\vec{n} \times(\vec{n} \times \vec{\beta})}{(1-\vec{\beta} \cdot \vec{n})}\right) \mathrm{d} t^{\prime} \\
& =\frac{e}{c R}\left[1 \cdot \frac{\vec{n} \times(\vec{n} \times \vec{\beta})}{(1-\vec{\beta} \cdot \vec{n})}\right]_{t_{0}^{\prime}}^{t_{1}^{\prime}}-\frac{e}{c R} \int_{t_{0}^{\prime}}^{t_{1}^{\prime}} 0 \mathrm{~d} t^{\prime} \\
& =\frac{e}{c R}\left(\frac{\vec{n} \times(\vec{n} \times \vec{\beta})}{(1-\vec{\beta} \cdot \vec{n})}\right)
\end{aligned}
$$

Likewise, one gains the electric field for the end-point of the trajectory:

$$
\int \vec{E}(\vec{x}, t) d t=-\frac{e}{c R}\left(\frac{\vec{n} \times(\vec{n} \times \vec{\beta})}{(1-\vec{\beta} \cdot \vec{n})}\right)
$$

It is important to first transform the integration time into retarded time before calculating the integral. The electric fields of the end-point 1 and of the radiation along the track then have to be added to get a self-consistent implementation for modelling radio emission in an air showers.

\section{Continuous vs. discrete calculation and incorporation of end- point contributions}

Adding the discrete endpoint contributions to the continuous contributions along the tracks may produce problems. The radiation associated with the end-points not only contains the emission due to the tangential acceleration. Due to the change in the direction of particle movement between the beginning and the end of the trajectory, radiation associated with the perpendicular acceleration, which was so far treated in the continuous description, is also contained. Combining the two descriptions therefore exhibits a risk of double-

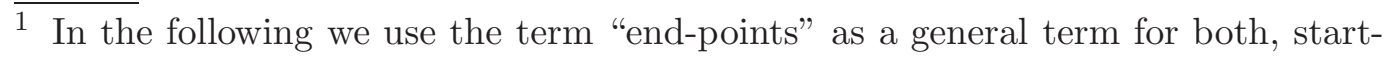
points and end-points, as both are treated in the exact same way. 
counting. In order to avoid such problems, it is preferable to change the calculation for the continuous contributions along the curved particle tracks to a discrete representation. Hence, the chosen representation in REAS3 is completly discrete to ensure that the calculations are self-consistent. To describe

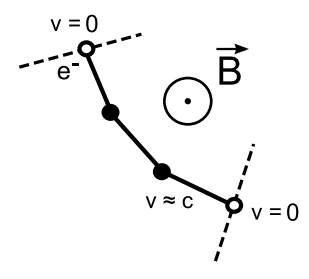

Fig. 3. Sketch of the trajectory with a discrete description and end-points.

radio emission contributions along the particle trajectories in a discrete picture, the trajectories of the particles are split in straight track fragments joined by "kinks" in which the velocity of the particles is changing instantaneously. A sketch of this description is given in figure 3. The instantaneous change of velocity at the kinks leads to radiation. With particle velocity $\overrightarrow{\beta_{1}} \cdot c$ before and $\overrightarrow{\beta_{2}} \cdot c$ after the kink, radiation for one kink of the trajectory is:

$$
\begin{aligned}
\int \vec{E}(\vec{x}, t) \mathrm{d} t & =\int_{t_{1}}^{t_{2}} \frac{e}{c}\left|\frac{\vec{n} \times[(\vec{n}-\vec{\beta}) \times \dot{\vec{\beta}}]}{(1-\vec{\beta} \cdot \vec{n})^{3} R}\right|_{r e t} \mathrm{~d} t=\vec{F}\left(t_{2}\right)-\vec{F}\left(t_{1}\right) \\
& =\frac{e}{c R}\left(\frac{\vec{n} \times\left(\vec{n} \times \overrightarrow{\beta_{2}}\right)}{\left(1-\overrightarrow{\beta_{2}} \vec{n}\right)}\right)-\frac{e}{c R}\left(\frac{\vec{n} \times\left(\vec{n} \times \overrightarrow{\beta_{1}}\right)}{\left(1-\overrightarrow{\beta_{1}} \vec{n}\right)}\right)
\end{aligned}
$$

To verify that the continuous and the discrete calculations of emission contributions along the trajectories are equivalent and that both descriptions produce the same results, the REAS2 code was changed to calculate emission using the discrete approach of straight track segments connected with kinks (without the additional contributions of end-points). Analytically, the equivalence of the two approaches for the frequency domain has been shown by Konstantinov et al. [20]. Several tests with the REAS code have confirmed that the implementation of the discrete description is equivalent to the continuous one as can be seen in figure 4. The figure illustrates the equivalence for a vertical proton-induced air shower with primary energy of $E_{p}=10^{17} \mathrm{eV}$. The observer position is $100 \mathrm{~m}$ north of the shower core. The advantage of the discrete calculation is the consistency of the description of emission contributions along the tracks and emission at the endpoints which is making the incorporation of radiation at the endpoints canonical. To complement the former implementation in the Monte Carlo code with the emission due to the variation of the number of charged particles, it is therefore convenient to use the discrete description. In the discrete picture, contributions at the beginning and the end of a track are just kinks with $\overrightarrow{\beta_{1}}=0$ and $\overrightarrow{\beta_{2}}=0$, respectively. This self-consistent emission model has been incorporated in REAS3, taking 


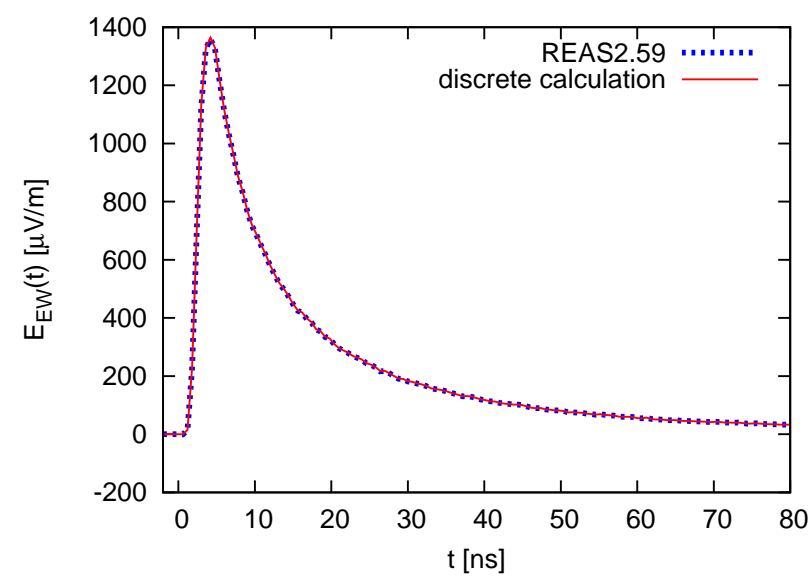

Fig. 4. Comparison of discrete and continuous description of radio emission (without endpoint contributions) for an observer $100 \mathrm{~m}$ north of shower core of a vertical $10^{17} \mathrm{eV}$ air shower. The results are identical.

the radiation at the beginning and the end of a trajectory as well as along the curved trajectory into account. The obtained results for the radio signal is discussed in section [6, but first the numerical stability will be demonstrated.

\section{$5 \quad$ Numerical stability}

As already mentioned in chapter 2, particle trajectories are represented by ensembles of several shorter, unrelated trajectories in the code. This ensures that the phase-space distribution of the analytically propagated particles stays consistent with the underlying particle distribution without having to treat energy losses during the propagation explicitly (cf. section 4 of [12]). The length of the short segments is controlled by a parameter $\lambda$. This parameter $\lambda$ determines the length of the short tracks into which the real trajectories of the particles are divided (it does not denote the sampling density of kinks on a track). If $\lambda$ is chosen inadequately large, the discrepancies between the particle distributions recreated in REAS and the distributions histogrammed in CORSIKA are getting too large. Thus, to avoid these discrepancies $\lambda$ has to be chosen small enough. In REAS2, it was recommended to set this parameter to $1 \frac{\mathrm{g}}{\mathrm{cm}^{2}}$, even though it is just a technical parameter, i.e. the result does not depend on the exact value of $\lambda$ as long as $\lambda$ is set small enough. Also, in REAS3 the tracklength of the single short trajectories should have no influence on the physics results as long as it is chosen small enough. This cross check was made for a vertical proton-induced air shower with $E_{p}=10^{17} \mathrm{eV}$. As shown in figure 5 for an observer $100 \mathrm{~m}$ north of the shower core, the result converges with decreasing values of $\lambda$. The same result is obtained for an observer $400 \mathrm{~m}$ north of the shower core which is displayed in the right column of figure 5. The comparison of both observer positions demonstrates that far away from the 
shower core, the result converges faster than close to the shower core. For $\lambda=$ $0.1 \frac{\mathrm{g}}{\mathrm{cm}^{2}}$, a stable result is obtained in both cases. For observer positions close to
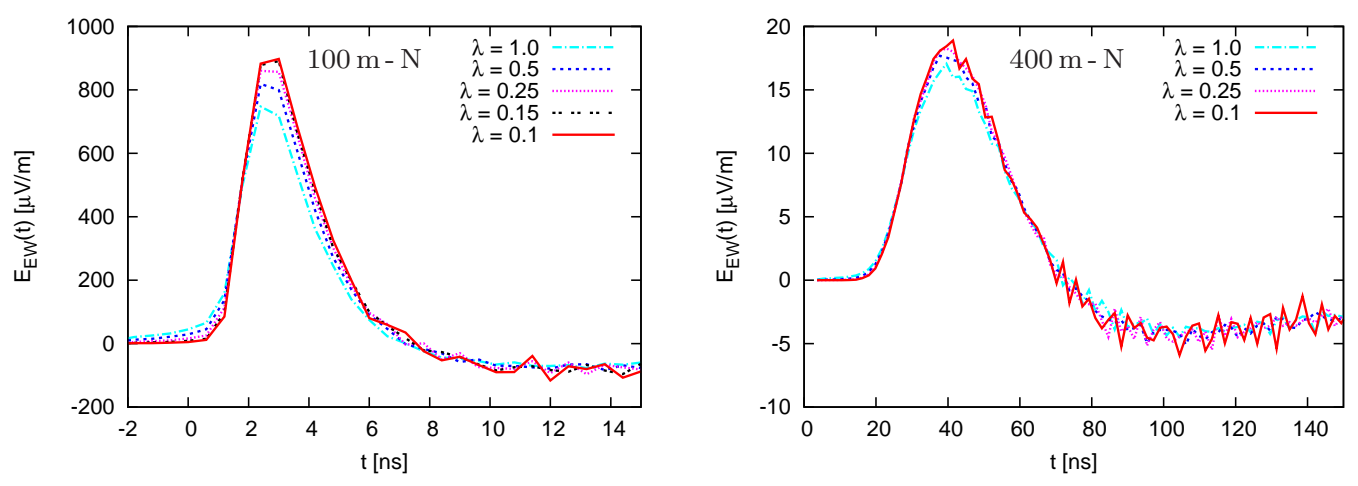

Fig. 5. Influence of different values of the technical parameter $\lambda$. Left: observer $100 \mathrm{~m}$ north of shower core. Right: observer $400 \mathrm{~m}$ north of shower core.

the shower core as for $100 \mathrm{~m}$ distance, it is also visible that for larger values of $\lambda$, e.g. for $\lambda=1.0 \frac{\mathrm{g}}{\mathrm{cm}^{2}}$, there is already a finite radio contribution for negative times. These unphysical contributions appear if partciles are created with symmetric trajectories around the place of particle generation and $\lambda$ is chosen inadequately large. For small enough values of $\lambda$, however, the symmetric trajectories ensure a faster convergence of the result than the asymmetric trajectories used in REAS2.

With smaller parameters of $\lambda$, however, the high frequency noise increases as well as the computing time. This first effect is larger for observers far away as can be seen in figure 5. To optimize the calculation in REAS3, the path depth $\lambda$ of the electron and positron trajectories is therefore chosen as a dynamical parameter depending on the lateral distance of the observer. Therefore, it is recommended to set $\lambda$ to $0.1 \frac{\mathrm{g}}{\mathrm{cm}^{2}}$ at the core and increase this value linearly every $100 \mathrm{~m}$ as it is done by default in REAS3. The advantage of this implementation is on the one hand to gain stable results for all observers and on the other hand to avoid high frequency noise for observers at larger lateral distances. The difference in the recommended value of $\lambda$ in REAS2 and REAS3 is due to the fact that the emission model in REAS3 requires a precise description of the particle momenta during the propagation, which requires a more fine-grained treatment.

\section{Results}

In chapter 5, we have verified that REAS3 is producing stable results and that the endpoints were implemented correctly. In chapter 6.1, we focus on the results obtained with REAS3 and in section 6.2 we discuss the influence of the charge excess in air showers on the radio emission. In both chapters, the radio emission was calculated for a proton-induced vertical air shower 
with primary energy of $E_{p}=10^{17} \mathrm{eV}$. The shower itself was generated with CORSIKA 6.7 and COAST. The positions of the observers were chosen at sea level with different lateral distances and relative observer orientations to the shower core. For the simulations with REAS2 and REAS3, identical showers were taken. This is easily possible because the histogramming approach allows an easy seperation between the air shower modelling and the radio emission calculation.

\subsection{REAS2 vs. REAS3}

To study the changes introduced by the implementation of emission due to the variation of the number of charged particles, a simple, vertical shower geometry was chosen as specified above. The magnetic field was taken as horizontal with a field strength of 0.23 Gauss to get a geomagnetic angle of $90^{\circ}$. Figure 6] shows a comparison between unfiltered (i.e. unlimited bandwidth) pulses and frequency spectra of REAS2 and REAS3 for observers with different positions with respect to the shower core. The spectra show the total field strength for two azimuthal observer directions: the thick line displays the total field strength for an observer east of the shower core and the thin line for an observer north of the shower core. It is clear that the strength of the pulses as well as the time structure of the pulses has changed, while the changes in the pulse amplitudes are dependent on the observer azimuth angle. The time structure of the pulses has changed from unipolar to bipolar. This can also be seen in the frequency spectra (lowest row of figure 6) where in case of REAS3, the spectral field strengths drop to zero for frequency zero, as it was the case in the analytical implementation [9]. It can be argued from basic physical arguments that the spectral field strength has to drop to zero for small frequencies because the source of the coherent emission exists only for a finite time in a finite region of space and thus the the zero-frequency component of the emission, which corresponds to an infinite time-scale, can contain no power (cf. [21]).

While the emission pattern of REAS2 was azimuthally asymmetric, REAS3 simulations are much more symmetric, as is expected for the given shower geometry. The increased symmetry can also be seen in the frequency spectra where the characteristics of the spectral field strengths with frequency are getting very similar for the two different azimuthal observer positions in case of REAS3. The remaining asymmetry will be discussed in section 6.2. To get a more general impression of the changes in the amplitudes of the signal with REAS3, the lateral dependence of the emission is studied. Figure 7 shows this dependence for the unfiltered, full bandwith amplitudes. In REAS2, there was a much stronger signal for observers in the north or south than for observers in the east or west. In REAS3, the signal pattern is much more symmetric, but obervers in the eastern direction measure a higher absolute field strength 

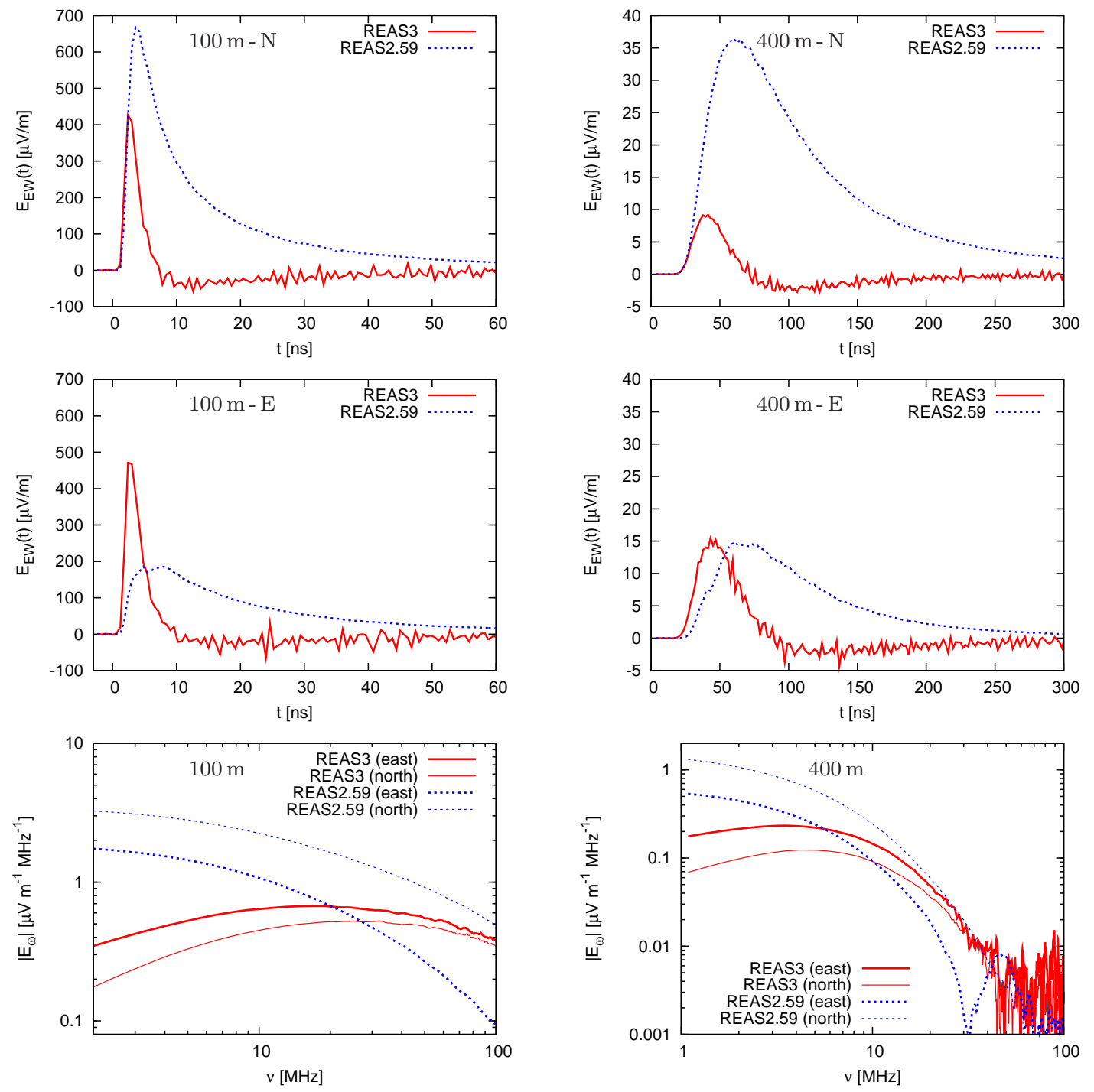

Fig. 6. Upper row: raw pulses for observers $100 \mathrm{~m}$ (left) and $400 \mathrm{~m}$ (right) north of shower core. Middle row: raw pulses for observers $100 \mathrm{~m}$ (left) and $400 \mathrm{~m}$ (right) east of shower core. Lowest row: frequency spectra for observers $100 \mathrm{~m}$ (left) and $400 \mathrm{~m}$ (right) east and north of shower core. For the raw pulses the east-west polarisation of the electric field is shown, whereas for the frequency spectra the total spectral field is shown.

and observers in the west see a lower field strength than observers with other azimuthal positions. In general, the amplitudes of the field strength got lower from REAS2 to REAS3, while the changes for observers north and south of the shower core are much larger than for observers in the east or the west. To compare simulations with experimental data (e.g. of LOPES), the REAS simulations have to be filtered to a finite observing bandwidth. This is done by the helper application REASPlot which is included in the REAS3 package. In this paper, REASPlot was used with an idealised rectangular $43 \mathrm{MHz}-76 \mathrm{MHz}$ bandpass filter which can lead to acausal contributions at negative times due 

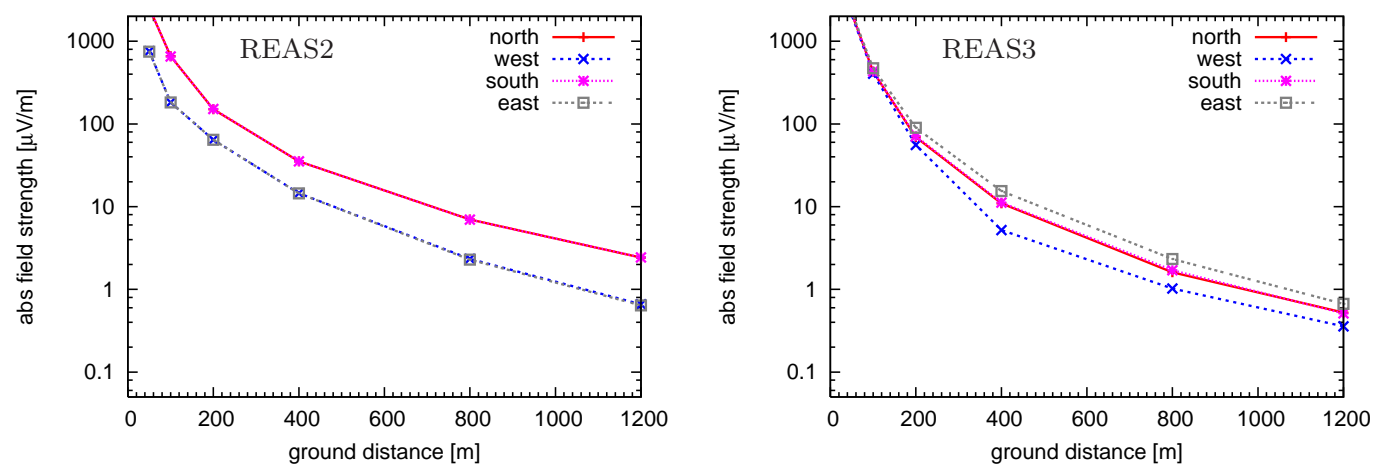

Fig. 7. Lateral dependence with full bandwidth amplitudes. Left: REAS2. Right: REAS3.

to the idealisation of the filter. In figure 8 , the filtered pulses for observers with lateral distance of $100 \mathrm{~m}$ east and north of the shower core are shown. The quick oscillations are determined by the selected filter bandwidth. The
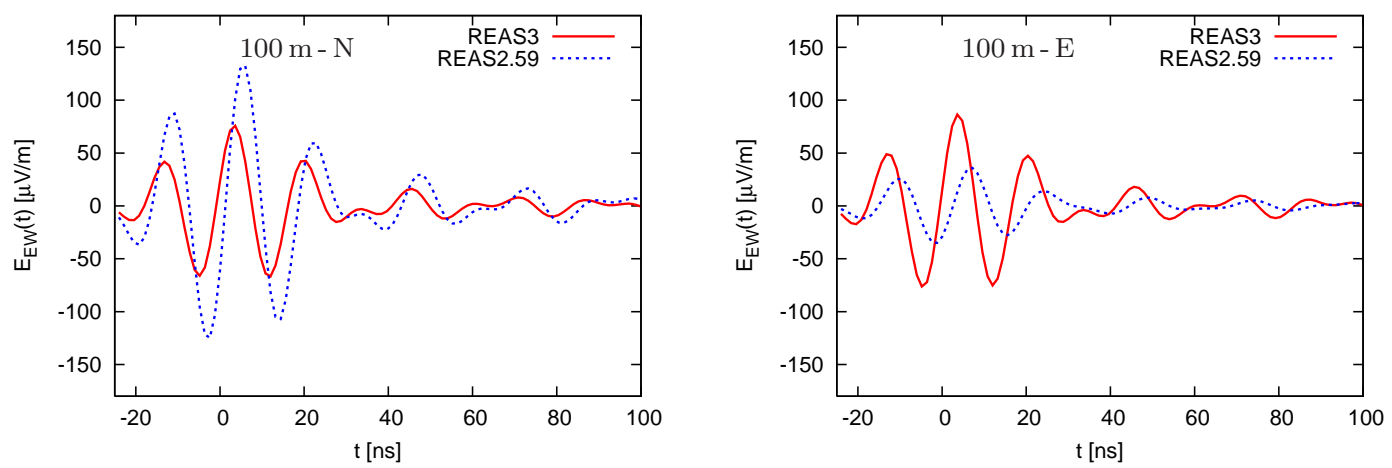

Fig. 8. Filtered pulses (a simple rectangular 43-76 MHz bandpass filter is used) for observers with $100 \mathrm{~m}$ distance to the shower core. Left: the observer is north of the shower core. Right: the observer is east of the shower core.

differences in the pulse strengths are much smaller for the filtered than for the raw pulses because the strongest changes between REAS2 and REAS3 occur at low frequencies (Please note that figure 6 is plotted on a log-log scale). In general, e.g. for different geometries, the differences in the amplitudes of the filtered pulses can be larger than in this example (cf. [22]).

Again, the increased azimuthal symmetry of REAS3 is observable. To get an overall impression of the change from REAS2 to REAS3 and the influence of the net charge excess on the east-west asymmetry, we show the contour plots of the $60 \mathrm{MHz}$ field strength in figure 9. The ellipticity which is seen in REAS2 is replaced by a nearly azimuthally symmetric pattern in REAS3. The "clover" pattern seen earlier in the north-south polarisation component is no longer visible. In the vertical polarisation component (not shown here), there is no significant flux for either of the two simulations, as expected for a vertical shower. In the contour plots of REAS3, the east-west asymmetry is visible as well. The resulting REAS3 emission pattern can be interpreted 
as a superposition of a circularly symmetric contribution with a $\vec{v} \times \vec{B}$ (in this case thus pure east-west) polarisation and a radially polarised emission contribution caused by the time-varying net charge excess.

In summary, the incorporation of radiation due to the variation of the num-
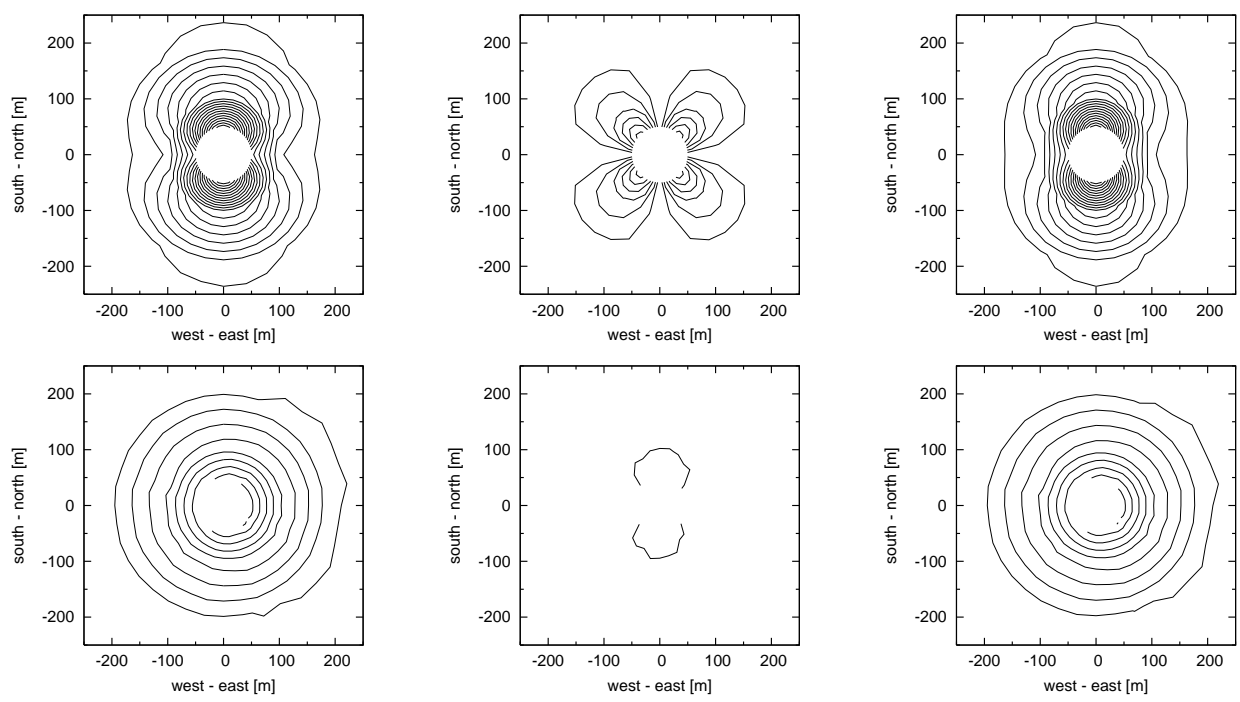

Fig. 9. Contour plots of the $60 \mathrm{MHz}$ field strength for emission from a $10^{17} \mathrm{eV}$ vertical air shower. From left to right: total field strength, north-south and east-west polarisation component. Contour levels are $0.1 \mu \mathrm{Vm}^{-1} \mathrm{MHz}^{-1}$ apart. The closest position of the simulated observers to the shower core is $50 \mathrm{~m}$. Upper row: REAS2. Lower row: REAS3

ber of charged particles in the form of endpoint contributions results in a clear change from REAS2 to REAS3. The revised, self-consistent implemented model in the Monte Carlo code REAS3 predicts bipolar pulses with a mostly symmetrical emission footprint. In addition to these changes, a further emission contribution arises from the variation of the net charge excess, which explains the remaining asymmetries. This contribution will be discussed in the following section.

\subsection{Discussion of charge excess emission}

The observed east-west asymmetry mentioned in section 6.1 arises from the fact that more electrons than positrons exist in an extensive air shower [23]. This net charge excess of order $10-20 \%$ leads to a contribution in the radio signal even in the absence of any magnetic field. Hence, in this section an air shower was simulated with CORSIKA for the geometry and primary characteristics as already mentioned at the beginning of this chapter, but in contrast to section 6.1, the magnetic field strength was set to 0 Gauss. Although the showers calculated with $\mathrm{B}=0.23$ Gauss and $\mathrm{B}=0$ Gauss do not represent the exact same particle distributions, we can interpret the predictions for the 
shower with $B=0$ Gauss as the contribution due to the net charge excess of the shower used in section 6.1. This allows us to test whether indeed the eastwest asymmetry can be associated with the pure charge excess. In REAS2, radio emission is produced due to deflection of charged particles in the magnetic field. Hence, there is no radiation without magnetic field. In contrast, REAS3 takes also emission due to the variation of the net charge excess into account. A radially polarised component for emission due to charge excess is expected, as seen also in the macroscopic approach [24]. The radiation pattern for a shower with $\mathrm{B}=0$ Gauss is indeed radially polarised as illustrated in the contour plots of the $60 \mathrm{MHz}$ field strength in figure 10, For the vertical component (not shown here) there is again no significant flux, as expected. Again, the closest observer position to the shower core is $50 \mathrm{~m}$. Please note that the contour levels for the simulation without magnetic field are smaller than for the simulation with magnetic field, i.e., the relative field strength of the charge excess emission at $60 \mathrm{MHz}$ is small in the distance range up to $200 \mathrm{~m}$. To study
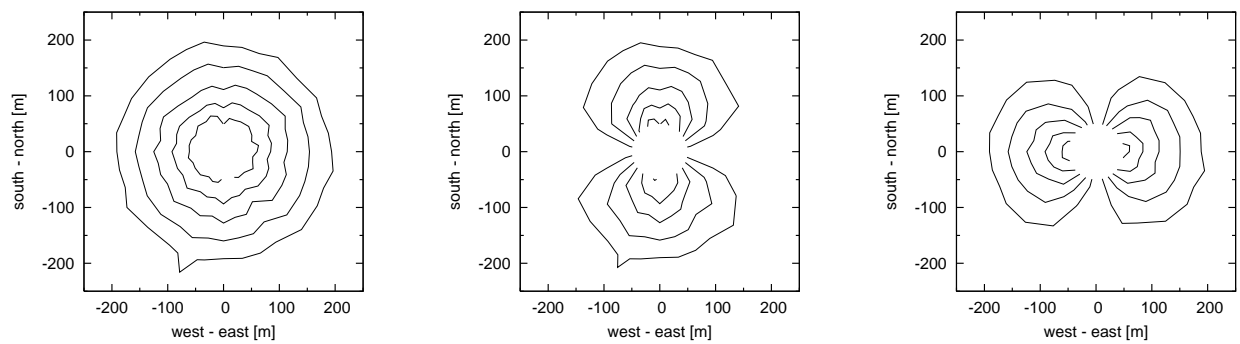

Fig. 10. Contour plots of the $60 \mathrm{MHz}$ field strength for emission from a $10^{17} \mathrm{eV}$ vertical air shower without any magnetic field. Contour levels are $0.03 \mu \mathrm{Vm}^{-1} \mathrm{MHz}^{-1}$ apart. The closest observer position to the shower core is $50 \mathrm{~m}$.From left to right: total field strength, north-south and east-west polarisation component. The "spike" in the lower-left part of the contours is associated with noise in the simulation.

the influence of the net charge excess emission on the overall radio signal, it is helpful to look at the polarisation vectors in the plane perpendicular to the shower axis. For the pure geomagnetic emission, the polarisation vectors at all observer positions point in the same direction as illustrated in the left sketch of figure 11. The right sketch illustrates the polarisation vector for the
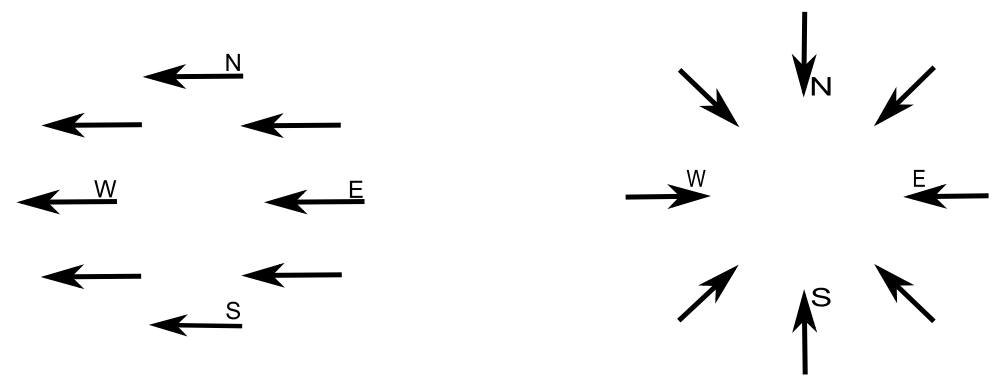

Fig. 11. Sketch of the polarisation vector. Left: uniform pattern as it is the case for pure geomagnetic emission. Right: radial pattern as it is the case for the net charge excess emission. 
emission due to the variation of the net charge excess. The direction in which the vector points is changing with the observer position, following a radial pattern. Hence, for an observer in the east the total signal $S_{E}$ is given by

$$
S_{E}=S_{g m}+S_{c e}
$$

where $S_{g m}$ is the pure geomagnetic contribution and $S_{c e}$ the net charge excess contribution to the signal. For an observer in the west, the total signal $S_{W}$ is composed of

$$
S_{W}=S_{g m}-S_{c e}
$$

With the signals measured east and west from the shower axis, the signal for the pure geomagnetic emission and for the net charge excess can be calculated by

$$
S_{g m}=\frac{1}{2}\left(S_{E}+S_{W}\right) \quad \text { and } \quad S_{c e}=\frac{1}{2}\left(S_{E}-S_{W}\right)
$$

To verify the assumption made in equations (5) and (6), we calculated the signal of the charge excess as it is resulting from above for the shower with $\mathrm{B}=0.23$ Gauss and compared this with the emission of the shower with $\mathrm{B}=0$ Gauss. Figure 12 illustrates that both pulses match. Therefore, the eastwest asymmetry in the azimuthal emission pattern seen in the figures of section
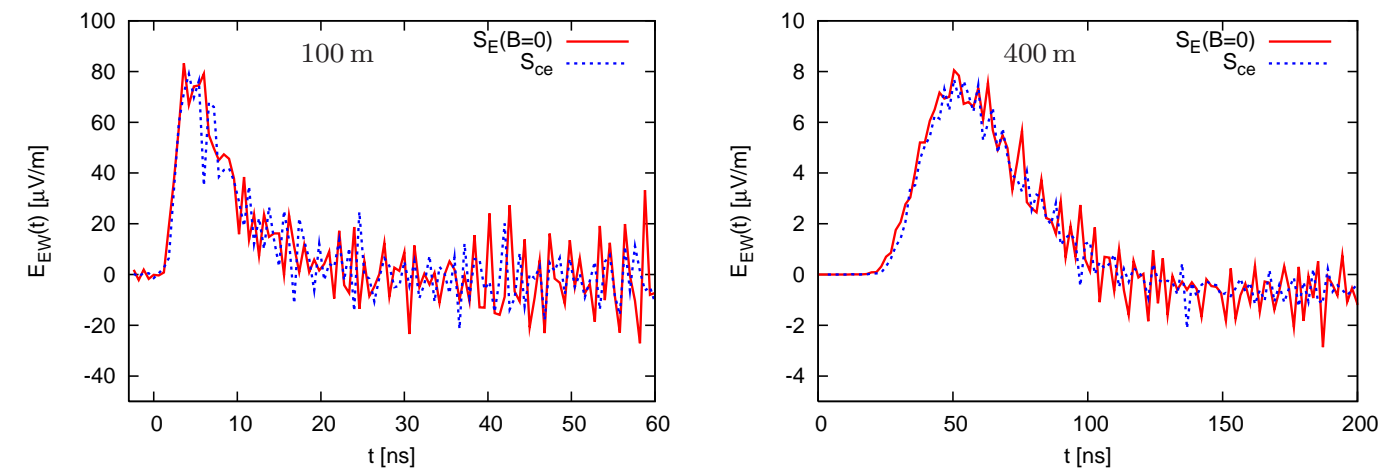

Fig. 12. Comparison of emission due to net charge excess without magnetic field (solid line) and the calculated signal for the net charge excess (dashed line) from a shower with magnetic field. Displayed is in each case the east-west polarisation. Left: $100 \mathrm{~m}$ distance from shower core. Right: $400 \mathrm{~m}$ from shower core.

6.1 is completely reducible to the emission of the net charge excess in an air shower.

Finally, it is interesting to quantify the relative strength of the charge excess emission with respect to the pure geomagnetic radio emission. Analyses studying the dependence of the radio signal on pure geomagnetic emission have already been done and have shown that there might be discrepancies between a pure geomagnetic model and the measured data [25]. The ratio of 
the net charge excess signal and the pure geomagnetic radiation can be calculated from equation (7) to quantify the relative influence of the net charge excess. Figure 13 illustrates the ratio for the unfiltered full bandwidth amplitudes and the 43 to $76 \mathrm{MHz}$ filtered bandwidth amplitudes for the east-west polarisation component. For the filtered case the ratio in the plot is shown only for observers up to $400 \mathrm{~m}$ lateral distance. The reason is that the frequency spectra for a vertical shower drop fast with increasing lateral distance as was also seen in figure 6. Consequently, in the used frequency range the signal is not anymore distinguishable from numerical noise at very large distances. For the amplitudes of the unfiltered pulses the charge excess has more and more influence with larger distances, from a few $\%$ close to the core to around $90 \%$ of the pure geomagnetic emission at $1200 \mathrm{~m}$. However, for the filtered pulses the ratio is almost constant over the whole range, at a level of $\sim 10 \%$. It is important to clarify that the emission of the net charge excess

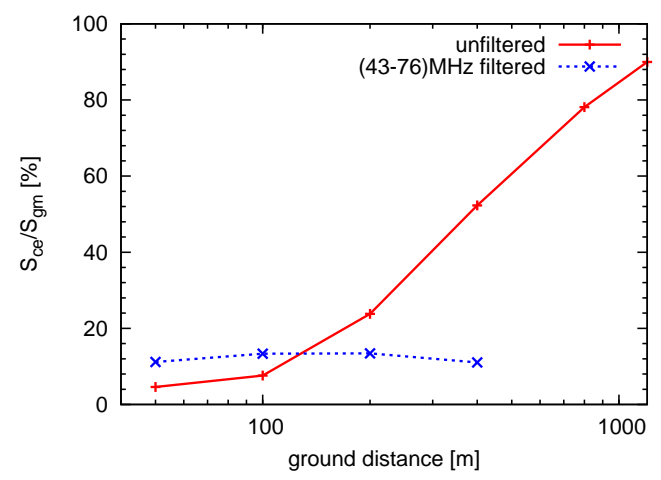

Fig. 13. Comparsion of the charge excess and the pure geomagnetic contribution on the radio signal of an vertical air shower for the east-west polarisation component. The lines may not represent the correct interpolation between the points due to the logarithmic scale of the x-axis. The ratio of the filtered data is only shown for distances up to $400 \mathrm{~m}$, because then the signal is not anymore distinguishable from noise in this frequency range.

occurs due to the variation of the number of charged particles and not due to Cherenkov-like emission. Both processes have been described in the pioneering articles of Askaryan [26], [27], but the term "Askaryan radiation" is today generally interpreted as Cherenkov emission in dense media. For the inclusion of Cherenkov-like emission, a refractive index is needed which is not "unity". REAS3 approximates the index of refraction to be unity so far.

\section{Conclusion}

Using an "end-point" formalism, we have implemented a fully consistent Monte Carlo model for radio emission from air showers, which in particular includes also the emission contributions due to the variation of the number of mov- 
ing charges. For the implementation into the simulation code REAS, the description was changed from a continuous treatment to a discrete description, because in the latter representation endpoint contributions can be added canonically. REAS3 produces stable results. The pulse shape has changed from unipolar pulses in REAS2 to bipolar pulses in REAS3. This is also apparent in the frequency spectra of REAS3 simulations which drop to zero for small frequencies. Several results illustrate the increased symmetry in the azimuthal emission pattern predicted by REAS3. Due to charge excess in extensive air showers it is evident that a small asymmetry has to remain. This effect was shown by calculating the pure geomagnetic and charge excess contributions from the asymmetries present in the full simulations and simulating radio emission in the complete absence of a magnetic field which agree well within numerical uncertainties. In the absence of a magnetic field, REAS3 predicts a radially polarized emission pattern. Due to the presence of this charge excess emission it is also obvious that the radio emission is not purely of geomagnetic origin and thus cannot be described by a pure $\vec{v} \times \vec{B}$ dependence, but that the signal polarisation depends on the exact observer position relative to the core. For a vertical shower, the relative strength of the emission due to the variation of the net charge excess with respect to the pure geomagnetic emission increases from a few $\%$ close to the core up to $\sim 90 \%$ at lateral distances of $1200 \mathrm{~m}$ for the unlimited bandwidth pulse amplitudes. This net charge excess affects the east-west symmetry as well as the polarisation of the radio emission. For the filtered $43 \mathrm{MHz}-76 \mathrm{MHz}$ bandwidth amplitudes the relative strength of the net charge excess with respect to the pure geomagnetic emission is constant at a level of approximately $10 \%$ for different lateral distances. Although the changes introduced with REAS3 are significant, many qualitative results obtained with earlier simulations are still valid (e.g., approximately exponential lateral distributions, a dependence of the lateral slope on $X_{\max }$, the coherent scaling of the pulse amplitudes, and the fall-off of the frequency spectra to higher frequencies and larger distances). Certainly, the absolute amplitudes, pulse shapes and values of scaling parameters have changed with REAS3. With the implementation of emission due to the variation of the number of charged particles in REAS3, for the first time a self-consistent timedomain model exists which takes the full complexity of air shower physics as provided by CORSIKA simulations into account. The source code of REAS3 will be freely available on request. REAS3 therefore constitutes a widely usable radio simulation tool which can be employed to study radio emission from air showers initiated by arbitrary primary particles, using any of the available hadronic interaction models, realistic atmospheric profiles and arbitrary shower geometries (including near-horizontal showers, since the atmosphere in REAS3 is treated with a curved geometry). 


\section{Acknowledgements}

The authors would like to thank S. Buitink, R. Engel, H. Falcke, O. Scholten and K.D.de Vries for very helpful discussions and A. Haungs for very useful comments to the manuscript. This research has been supported by grant number VH-NG-413 of the Helmholtz Association.

\section{References}

[1] H. Falcke et al. - LOPES Collaboration, Nature 435 (2005) 313-316.

[2] T. Huege et al. - LOPES Collaboration, Proc. of the ARENA conference 2010, Nantes, France, arXiv:1009.0345.

[3] D. Ardouin et al. - CODALEMA Collaboration, Nuclear Instruments and Methods in Physics Research A 555 (2005) 148-163.

[4] D. Ardouin et al. - CODALEMA Collaboration, Astroparticle Physics 31 (2009) 192-200.

[5] A.M. van den Berg et al. - Pierre Auger Collaboration, Proceedings of the $31^{\text {st }}$ International Cosmic Ray Conference (2009) \#0232 arXiv.org:0908.4422.

[6] J. Abraham et al. - Pierre Auger Collaboration, Nuclear Instruments and Methods in Physics Research A 523 (2004) 50.

[7] H.J.A. Röttgering, New Astronomy Review 47 (2003) 405.

[8] T. Huege - Proc. of the ARENA 2008 conference, Rome, Italy, Nuclear Instruments and Methods in Physics Research A 604 (2009) 57-63.

[9] T. Huege, H. Falcke, Astronomy \& Astrophysics 412 (2003) 19-34.

[10] T. Huege, H. Falcke, Astronomy \& Astrophysics 430 (2005) 779-798.

[11] T. Huege, H. Falcke, Astroparticle Physics 24 (2005) 116.

[12] T. Huege, R. Ulrich, R. Engel, Astroparticle Physics 27 (2007) 392-405.

[13] D. Heck et al., FZKA Report 6019, Forschungszentrum Karlsruhe (1998).

[14] O. Scholten, K. Werner, F. Rusydi, Astroparticle Physics 29 (2008) 94-103.

[15] F.D. Kahn, I. Lerche, Proc. Royal Soc. London A289, (1966) 206.

[16] M.A. Duvernois, B. Cai, D. Kleckner, Proc. of the 29th ICRC, Pune, India (2005) 311.

[17] D.A. Suprun, P.W. Gorham, Astroparticle Physics 20 (2003) 157-168. 
[18] T. Huege, M. Ludwig, O. Scholten, K.D. deVries, Proc. of the ARENA conference 2010, Nantes, France, arXiv:1009.0346.

[19] C.W. James, H. Falcke, T. Huege, M. Ludwig, submitted to Physical Rev. E (2010) arXiv:1007.4146v1.

[20] A.A. Konstantinov, PhD thesis, Lomonosov Moscow State University (2009).

[21] O. Scholten, K. Werner - Proc. of the ARENA 2008 conference, Rome, Italy, Nuclear Instruments and Methods in Physics Research A 604 (2009) 24-26.

[22] M. Ludwig, T. Huege - Proc. of the ARENA conference 2010, Nantes, France, arXiv:1009.1994 1 .

[23] T. Bergmann, R. Engel, D. Heck et al., Astroparticle Physics 26 (2007) 420-432.

[24] K. Werner, O. Scholten, Astroparticle Physics 29 (2008) 393-411.

[25] P.G. Isar et al. - LOPES Collaboration, Proc. of the 31st ICRC, Lodz, Poland (2009) 1128.

[26] G.A. Askaryan, Soviet Physics, JETP 14 (1962) 441.

[27] G.A. Askaryan, Soviet Physics, JETP 21 (1965) 658. 